\title{
Propuestas para garantizar modalidades de consumo y producción sostenibles (ODS I2)'
}

\section{Mónica García Goldar²}

Resumen: En este trabajo partimos del contexto social actual (sobreconsumismo, obsolescencia programada, etc.) para ilustrar la escasa orientación de ciertas normas del Derecho europeo de consumo hacia la sostenibilidad; hecho que contrasta con la aprobación por parte de las instituciones europeas de dos planes de acción para la economía circular (en 2015 y en 2020) que no se han visto reflejados en la reciente Directiva 2019/771, de 20 de mayo, sobre determinados aspectos de los contratos de compraventa de bienes. Por fortuna, y a pesar de que esta Directiva es de armonización máxima, se concede un cierto margen de maniobra en favor de los Estados miembros, que podrán establecer periodos más largos de responsabilidad, como así sería lo deseable. Por último, también haremos referencia a la posibilidad de que el mercado se vaya autodirigiendo hacia la circularidad, toda vez que existe en la actualidad una mayor demanda por productos sostenibles.

Palabras clave: sobreconsumismo; obsolescencia; economía circular; garantías; productos; sostenibilidad.

Fecha de recepción: I5 de marzo de $2021^{3}$.

Fecha de admisión definitiva: I5 de abril de 202I.

'Primer Premio en la segunda edición del Premio Universidad Loyola a la Investigación en Desarrollo.

${ }^{2}$ Universidad de Santiago de Compostela.

${ }^{3}$ Fecha de cierre de la convocatoria de la segunda edición del Premio Universidad Loyola a la Investigación en Desarrollo. 
Proposals to guarantee sustainable consumption and production models (SDG 12)

Abstract: This work takes the current social context (overconsumption, programmed obsolescence, etc.) as a starting point in order to illustrate the lack of focus on sustainability reflected in certain European legal standards; a fact that contrasts with the approval by European institutions of two action plans for the circular economy (in 2015 and 2020) that were not reflected in the recent Directive 2019/771, May 20th, which regulates certain aspects concerning contracts for the sale of goods. Fortunately, and despite the fact that this Directive is geared toward maximum harmonisation, member countries will retain a certain margin for manoeuvre that will allow them to establish longer liability periods, which is desirable. Lastly, we will contemplate the possibility of the market moving towards circularity, as demand for sustainable products is growing.

Key words: overconsumption; obsolescence; circular economy; warranties; products; sustainability.

\section{Introducción}

El 25 de septiembre de 2015, la Asamblea General de Naciones Unidas aprobó la Resolución "Transformar nuestro mundo: la Agenda 2030 para el Desarrollo Sostenible" en la que se contemplan un total de 17 objetivos, todos ellos de gran trascendencia, como poner fin a la pobreza (objetivo 1), lograr la igualdad de género (objetivo 5) o reducir la desigualdad en los países y entre ellos (objetivo 10). También importante es el objetivo número 12, relativo a garantizar modalidades de consumo y producción sostenibles. Pues bien, el cometido de las páginas que siguen es mostrar la necesidad de que dichos objetivos se acompañen de medidas legislativas reales en materia de consumo, ya que de nada sirve informar y empoderar a los personas para que adopten pautas de consumo más sostenibles
Propositions pour garantir des modèles de consommation et production durables (ODD 12)

Résumé: Dans ce travail nous partons du contexte social actuel (surconsommation, obsolescence programmée, etc.) pour illustrer la faible orientation de certains règlements du droit européen de la consommation vers la durabilité; un fait qui contraste avec l'approbation par les institutions européennes de deux plans d'action pour l'économie circulaire (en 2015 et en 2020), qui n'ont pas été prises en compte dans la récente directive 2019/771 du 20 mai 2019 sur certains aspects des contrats de vente de biens. Heureusement, et malgré le fait que cette directive soit d'harmonisation maximale, une certaine marge de manœuvre est accordée aux États membres, qui pourront établir des périodes de responsabilité plus longues, comme il serait souhaitable. Enfin, nous évoquerons également la possibilité que le marché évolue vers la circularité en raison d'une demande croissante de produits durables.

Mots clé: surconsommation, obsolescence, économie circulaire, garanties, produits, durabilité. 
(sub-propósito 12.8) si, por ejemplo, el plazo de garantía para los bienes de consumo (incluso los duraderos) sigue siendo tan exiguo que obliga a los consumidores a que, una vez superado aquel, compren otro bien cuando el que tienen deja de funcionar correctamente.

\section{El contexto actual: sociedad sobreconsumista y obsoles- cencia programada}

El Nuevo Plan de acción para la economía circular por una Europa más limpia y más competitiva (2020) comienza con una demoledora sentencia: "Sólo tenemos una Tierra, pero en 2050 el consumo mundial será el equivalente al de tres planetas". Es difícil determinar en qué momento exacto nos convertimos en la denominada "sociedad de consumo"; proceso que Bauman (2006) describe como paso de la sociedad de productores a la sociedad de consumidores. Además, este cambio no se redujo únicamente a la (mayor) cantidad de productos o servicios consumidos, sino también, y lo que es quizá peor, a la (peor) calidad de lo que consumimos. Tras la Revolución Industrial y hasta principios del siglo XX, los fabricantes solían producir objetos para satisfacer nuestras necesidades; objetos cuya cualidad inherente era la durabilidad -cuanto más resistente al paso del tiempo era un producto, mayor era la valoración por parte de los consumidores-. Muy a la inversa, hoy en día se generan necesidades que sólo se satisfacen con determinados productos los cuales son, a su vez, mucho menos duraderos (Norma Martínez y Margarita Porcelli, 2016, 336-339). Esta nueva jerarquía de valores, heredada del denominado "síndrome consumista", ha depreciado la durabilidad y sobrevalorado la fugacidad; además, ha hecho que la apropiación, seguida de una rápida eliminación de los residuos, sea la premisa de la que parte, exaltando la rapidez, el exceso y el desperdicio (Bauman, 2006).

He aquí el principal problema: el consumo se ha transformado en consumismo. Ambos conceptos no tienen nada que ver: el consumo es la acción destinada a satisfacer las necesidades inmediatas del ser humano y el consumismo es el consumo de productos no necesarios y rápidamente sustituibles por otros (Ribeiro Freyesleben y Maas Dos Anjos, 2018, 812). Si esto sólo tuviese repercusiones (positivas) en el crecimiento económico, no habría razones para alarmarse; el problema es que el consumismo exacerbado degrada el medio ambiente (Norma Martínez y Margarita Porcelli, 2016, 337). Pues bien, dentro de las posibles causas que explicarían este cambio social se encuentra la introducción de ciertas prácticas comerciales deshonestas como la obsolescencia programada. 
La obsolescencia programada es un término bastante auto-descriptivo: se trata de la pérdida de funcionalidad de un producto (obsolescencia) que se introduce en el diseño de forma intencional (programada), para que la vida útil se reduzca considerablemente; se trata, pues, de una caducidad deliberada (Norma Martínez y Margarita Porcelli, 2016, 341). El momento de su aparición se remonta a comienzos del siglo $X X$, cuando un agente inmobiliario propuso su introducción para terminar con la crisis de la Gran Depresión, y en 1954 alcanzó su mayor popularidad, cuando Brooks Stevens dio una conferencia sobre la nueva producción en masa (Norma Martínez y Margarita Porcelli, 2016, 342). Su finalidad es eminentemente económica, pues consiste en

asegurar que los consumidores acudan al mercado una y otra vez a adquirir productos semejantes más actuales y renovados que presten la misma funcionalidad -o una similar-, al observar que aquellos que ya poseen, han devenido obsoletos. Las empresas logran así, mediante este estímulo singular, incrementar la tasa de reposición de los bienes que producen y aumentar los beneficios de todas las industrias (Soto Pineda, 2015, 3).

El sociólogo Packard distinguió en The waste makers (1960) hasta tres tipos de obsolescencia. En primer lugar, la obsolescencia de calidad o planificada es aquella que reduce deliberadamente la vida útil del producto, que se proyecta para deteriorarse en un tiempo menor del que llevaría normalmente. En segundo lugar, la obsolescencia funcional es la que se da cuando un producto queda desfasado porque se introduce otro en el mercado que funciona mejor. Por último, la obsolescencia de deseabilidad es la que se produce en nuestras mentes cuando, a pesar de que el producto sigue funcionando correctamente, pasamos a considerarlo anticuado. En la actualidad, y debido al proceso de digitalización en que nuestra sociedad se halla inmersa, se han distinguido dos nuevos tipos de obsolescencia: el primero sería la obsolescencia por incompatibilidad o informática, como es el caso, por ejemplo, de un programa informático que deja de funcionar al actualizarse el sistema operativo; el segundo, la obsolescencia indirecta, que deriva de la imposibilidad de reparar un producto por falta de piezas de recambio adecuadas o por resultar imposible la reparación (Libaert \& Haber, 2014).

De los cinco tipos de obsolescencia que existen, quizás el menos preocupante sea la obsolescencia técnica o funcional, siempre y cuando haga referencia a una mejora sustancial (y no meramente superflua o visual) del producto. En este sentido, cabe traer a colación el estudio de Vidalenc y Meunier (2014, 14-17), que al analizar si los productos de consumo más duraderos son mejores para el medio ambiente, los clasifican en dos categorías: en primer lugar estarían los productos que generan un $80 \%$ del impacto ambiental en la etapa de su utilización (por ejemplo los electrodomésticos) y en segundo lugar, los productos que generan un $80 \%$ del impacto ambiental en la etapa de su fabricación, como por ejemplo la 
vestimenta, los ordenadores o los smartphones. Si una nueva tecnología permite economizar el gasto energético anual de ese producto, sería interesante acelerar el recambio y no alargar la vida de ese producto; por lo tanto, la obsolescencia técnica puede ser, en algunos casos, beneficiosa para el medio ambiente. Con igual criterio, entendemos que evitar la obsolescencia informática podría no sólo resultar complicado, sino también arriesgado, si con ello dejamos de beneficiarnos de las ventajas que ciertos tipos de progreso pueden representar para nuestro día a día.

Más perniciosos parecen los otros tres tipos de obsolescencia. La obsolescencia de calidad, prematura o propiamente planificada presenta varias aristas y preocupa desde hace años -por sus efectos nocivos al ambiente- a economistas, sociólogos, ingenieros y juristas. Desde luego, existen motivos más que fundados para que este tipo de obsolescencia genere preocupación, y por ello, no debe extrañar que se empiecen a tomar medidas serias para erradicar esta práctica comercial tan extendida. Destacan, en este sentido, las medidas adoptadas en Francia en contra de la obsolescencia programada, donde se tipifica como delito (por todos, Santamaría Arinas, 2019, 3).

Otro tipo de obsolescencia que también debe alarmanos es la obsolescencia estética, psicológica o de deseabilidad, y que es más difícil (por no decir imposible) de sancionar. Parece que ya Packard supo ver, en The waste makers (1960), que tal vez era esta forma de obsolescencia la que más debía preocupar, pues a ella le dedicó un capítulo entero de su obra. Para este autor no cabe duda de que la sensación de desapego que se genera en algunos consumidores proviene de los propios comerciantes, que a menudo incorporan pequeños cambios de estilo para que aquellos sientan la necesidad de tener la más reciente versión. La obsolescencia psicológica se interrelaciona de forma acusada con el fenómeno social de las modas: en la moda, como en la modernidad, lo nuevo es preferido a lo viejo. Una de las funciones de la moda es precisamente atribuir a los objetos un valor simbólico que, adecuado a las referencias culturales de la sociedad, permite al consumidor la afirmación de su personalidad (Mesacasa, Corona y Mello, 2010, 2-4). Por ello, la obsolescencia psicológica es común en la industria de la moda, pero las principales empresas de tecnología, imitando a aquella, llevan años produciendo modelos nuevos con algún añadido que aparentemente los hace más novedosos (Norma Martínez y Margarita Porcelli, 2016, 347; Soto Pineda, 2015, 3). En esta tesitura, los consumidores no deben ser vistos como agentes responsables, sino como un colectivo social que debe ser tutelado por el ordenamiento jurídico; como dicen Pantzar \& Suljada $(2020,13)$, es difícil hacer responsables a los consumidores del cambio transformacional mientras las palancas del mercado y de la sociedad sigan incentivándoles a incrementar sus niveles de consumo. 
Por último (pero no por ello menos alarmante), la obsolescencia indirecta también repercute negativamente en el sobreconsumismo, haciendo muy difícil o casi imposible la reparación de ciertos productos. Un claro ejemplo lo encontramos en la multinacional Apple cuya política empresarial se ha visto cuestionada desde 2001 por ser contraria a la protección de consumidores, la defensa de la competencia y la protección medioambiental. Soto Pineda $(2015,4)$ trae a colación algunas de sus conductas más problemáticas, como las modificaciones que la multinacional hizo de los tornillos de algunos de sus productos o los adaptadores de corriente, que son modificados de forma reiterada; también menciona el asunto Westley vs. Apple Computer Inc (año 2003), relativo a la duración limitada de las baterías de litio de los IPod de las dos primeras generaciones y la imposibilidad de refacción de estos.

Como se puede ver, existen muchas formas de obsolescencia, aunque la más conocida es la programada; sin embargo, y aunque la percepción sobre ella es manifiesta y genera rechazo, lo cierto es que crea en los consumidores cierto nivel de vacilación y resignación, pues interpretan que es una realidad empresarial indubitada ante la cual son pocas las acciones de hecho y de derecho que se pueden ejercer (Soto Pineda, 2015, 2). Esto, por fortuna, no es cierto. Existe una alternativa posible: el consumo sostenible, que precisa de un nueva figura de consumidor: responsable, solidario, consciente de sus acciones y de la posibilidad de incidir en los hábitos sociales, económicos y ambientales. El consumo sostenible es a la vez consumo crítico y consumo ético (Norma Martínez y Margarita Porcelli, $2016,366)$, y representa uno de los ejes fundamentales de la economía circular, basada en el principio de nada perder y todo transformar, en clara contraposición a la economía lineal, basada en la línea de producción: extraer, producir, usar y tirar (De Boeck, 2019, 185; Mak \& Terryn, 2020, 229).

La economía circular es el único módelo que tiene sentido para el futuro a largo plazo (Schally, 2019, 5), pues se concibe como proyecto multi-R: repensar, rediseñar, reestructurar, reparar, redistribuir, reducir, reutilizar, reciclar, recuperar energía (Ribeiro Freyesleben y Maas Dos Anjos, 2018, 825-826). Es un concepto que la Unión Europea maneja al menos desde 2005 y que ha dado lugar a más de cien definiciones (Santamaría Arinas, 2019, 3), pero que viene a ser el resultado de los discursos que emergieron en años recientes y que pretenden lograr sinergias "win-win" entre el crecimiento económico y la protección medioambiental (Hobson, 2013, 1085).

La importancia de esta transición económica es tal que las instituciones europeas aprobaron dos planes de acción para hacerla realidad: el primero, a finales de 
2015, titulado "Cerrar el círculo: un plan de acción de la UE para la economía circular", en el que se abordaba, por fin, la necesidad de transitar hacia un modelo de crecimiento económico sostenible. En dicho documento se establecieron las bases que regirían la transformación, abarcando todo el proceso relativo al diseño/producción, consumo y gestión de residuos, e incluyendo medidas específicas para ciertas áreas prioritarias (plásticos, materias primas críticas, etc.). En lo que a consumo se refiere, el plan consideraba, muy acertadamente, que las decisiones de millones de consumidores podrían repercutir en apoyo o detrimento de la economía circular; decisiones que se toman con base en diversos criterios, como la variedad, el precio y la información a la que se tiene acceso. Casi cinco años después, en marzo de 2020, la Comisión lanzó el "Nuevo Plan de acción para la economía circular por una Europa más limpia y más competitiva", y que sigue, a grandes rasgos, el mismo esquema que el anterior Plan. Entre sus objetivos, se encuentran la transformación de las pautas de consumo para evitar que se produzcan residuos, mejorar la durabilidad, reutilizabilidad, actualizabilidad y reparabilidad de los productos, así como limitar el uso de productos de un solo uso y contrarrestar la obsolescencia programada. Se considera esencial empoderar a los consumidores y brindarles oportunidades de ahorro, garantizando que estos reciban información viable sobre la vida útil y sobre la disponibilidad de servicios de reparación, piezas de recambio y manuales de reparación. Pero, como dijimos, todos estos propósitos necesitan de medidas y cambios reales en la legislación de consumo, para no correr el riesgo de quedar en una mera declaración de intenciones. Pasamos, pues, a analizar en qué medida se han incorporado estos objetivos de sostenibilidad a la reciente Directiva (UE) 2019/771, de 20 de mayo, relativa a determinados aspectos de los contratos de compraventa de bienes, por la que se deroga la Directiva 1999/44/CE.

\section{El régimen de conformidad en la compraventa de bienes de consumo}

\section{I. La transición de la Directiva 1999/44/CE a la Directiva (UE) 2019/77I}

El régimen de garantías en la compraventa de bienes de consumo se asienta actualmente sobre las bases establecidas en la Directiva 1999/44/CE. A grandes rasgos, esta Directiva establece la obligación a cargo del vendedor de entregar al consumidor un bien que sea conforme con el contrato de compraventa (artículo 2); es decir, que se ajuste a la descripción realizada por el vendedor y sea apto para 
el uso especial requerido por el consumidor (conformidad subjetiva), y también que presente la calidad y las prestaciones habituales de un bien del mismo tipo y sea apto para los usos a que ordinariamente se destinen los bienes del mismo tipo (conformidad objetiva). En caso de falta de conformidad, el artículo 3 determina cuáles son los derechos del consumidor, que podrá: en primer lugar, exigir al vendedor que repare el bien o que lo sustituya sin cargo alguno, en un plazo razonable, y sin mayores inconvenientes para el consumidor, salvo cuando ello resulte imposible o desproporcionado; en segundo lugar, solicitar una reducción adecuada del precio o la resolución del contrato.

El plazo por el que el vendedor resulta responsable por la falta de conformidad es de dos años según el artículo 5; precepto que también indica que, salvo prueba en contrario, se presumirá que las faltas de conformidad que se manifiesten en un período de seis meses a partir de la entrega del bien ya existían en esa fecha. La extensión de ambos plazos es bastante exigua, pero como bien dice Mazeika $(2019,14)$, el marco legal que se estableció en la Directiva 1999/44/CE nunca pretendió promover los objetivos de la economía circular, pues por aquel entonces, dicha noción apenas existía.

Debe destacarse, de otra parte, que el objetivo de esta Directiva no era unificar, sino simplemente

aproximar las disposiciones legales, reglamentarias y administrativas de los Estados miembros sobre determinados aspectos de la venta y de las garantías de los bienes de consumo, con el fin de garantizar un nivel mínimo uniforme de protección de los consumidores en el marco del mercado interior (artículo 1).

Se trata, pues, de una Directiva de armonización mínima, que permite a los Estados miembros adoptar o mantener "disposiciones más exigentes, compatibles con el Tratado, para garantizar al consumidor un nivel de protección más elevado" (artículo 8). En el proceso de transposición de esta Directiva, algunos Estados miembros decidieron hacer uso de esta opción, estableciendo un régimen de garantías más favorable para el consumidor, pero las progresivas divergencias que se fueron observando pasaron a representar, con el tiempo, un obstáculo al mercado interior. Como consecuencia de ello, y veinte años después, se aprobó la Directiva (UE) 2019/771, que vino a derogar la Directiva 1999/44/CE. La principal razón que se adujo para la revisión de la normativa se encuentra en los considerandos 6 a 8 , y no es otra que la referida fragmentación jurídica, ya que las disposiciones nacionales que transpusieron la Directiva 1999/44/CE "difieren significativamente en la actualidad en cuanto a los elementos esenciales, como la ausencia o la existencia de una jerarquía entre las medidas correctoras". Dispa- 
ridades que pueden afectar negativamente a las empresas y a los consumidores: a las empresas, porque los costes adicionales que implican podrían llevarlas a preferir seguir operando a nivel nacional, perdiendo oportunidades para la expansión comercial; a los consumidores, porque la fragmentación también afecta negativamente a sus niveles de confianza en el comercio transfronterizo, por la incertidumbre respecto de sus derechos contractuales.

Esta situación se ha intentado corregir por la nueva Directiva (UE) 2019/771 a través de un nivel de armonización máximo, tal y como se establece en el artículo 4 :

Los Estados miembros no podrán mantener o introducir, en su Derecho nacional, disposiciones que se aparten de las establecidas en la presente Directiva, en particular disposiciones más o menos estrictas para garantizar un diferente nivel de protección de los consumidores, salvo que se disponga de otro modo en la presente Directiva;

nótese, sin embargo, que son varias las excepciones a este propósito de uniformidad.

\subsection{La Directiva (UE) $2019 / 77$ y el novedoso criterio de la durabilidad}

La Directiva (UE) 2019/771, de 20 de mayo, relativa a determinados aspectos de los contratos de compraventa de bienes (en adelante, DCB) todavía está en proceso de transposición por parte de los Estados miembros, que podrán hacerlo hasta el 1 de julio de 2021 (aunque las normas de transposición no serán aplicables hasta el 1 de enero de 2022); pero ello no nos impide analizar si esta actualización sobre las garantías legales es más compatible con los objetivos de sostenibilidad. Una de las primeras cuestiones a destacar es que la nueva Directiva introduce un prometedor criterio de conformidad no contemplado en la anterior Directiva 1999/44/CE: la durabilidad. Este concepto se define en el artículo 2 apartado 13 como "la capacidad de los bienes de mantener sus funciones y rendimiento requeridos en condiciones normales de utilización". Dicha definición es bastante escueta en nuestra opinión, por cuanto no hace mención alguna al paso de tiempo; más adecuado sería considerar que la durabilidad es la capacidad de los bienes de mantener sus funciones (...) por el periodo de tiempo que razonablemente el consumidor pueda esperar, en atención a las concretas características del bien.

\section{En el considerando 32 de la Directiva se especifica que}

la durabilidad debe referirse a la capacidad de los bienes de mantener sus funciones y rendimiento obligatorios en condiciones normales de utilización. Para que los bienes sean conformes deben poseer la durabilidad que sea habitual en bienes del mismo tipo y que el consumidor pueda razonablemente esperar habida cuenta de la naturaleza de los bienes específicos (...). 
Como se puede observar, tampoco se hace mención en este considerando al criterio temporal, aunque sí se indica que para evaluar la durabilidad esperable se tendrán en cuenta "todas las demás circunstancias pertinentes, como el precio de los bienes y la intensidad o frecuencia del uso que el consumidor haga de estos". Es en este considerando 32 en el que, además, se hace referencia expresa a los objetivos de sostenibilidad:

Garantizar una mayor durabilidad de los bienes es importante para lograr patrones de consumo más sostenibles y una economía circular. De forma similar, resulta esencial mantener los productos no conformes fuera del mercado de la Unión reforzando la vigilancia del mercado y ofreciendo incentivos adecuados a los operadores económicos para aumentar la confianza en el funcionamiento del mercado interior. Con tales fines, una legislación de la Unión específica sobre productos es el medio más apropiado para introducir requisitos de durabilidad y otros requisitos relativos a los productos en relación con tipos o grupos específicos de productos, utilizando criterios adaptados a esa finalidad.

Inmediatamente a continuación, y dando sentido a la afirmación precedente, sobre la idoneidad de la normativa europea sobre productos, se determina que

La presente Directiva, por tanto, debe ser complementaria a los objetivos perseguidos en dicha legislación de la Unión específica sobre productos y debe incluir la durabilidad como criterio objetivo para la evaluación de la conformidad de los bienes. (...) Además, en la medida en que la información específica sobre la durabilidad se indique en cualquier declaración precontractual que forme parte de los contratos de compraventa, el consumidor debe poder confiar en ella como parte de los criterios subjetivos de conformidad.

Por lo tanto, el considerando 32 DCB determina que la durabilidad puede constituir, según el caso, un criterio subjetivo, y constituirá, siempre y en todo caso, un criterio objetivo de conformidad. Esta premisa se corrobora con la lectura del artículo 7 que, al determinar los criterios objetivos, exige en su apartado d) que los bienes de consumo presenten la calidad y posean

las cualidades y otras características, en particular respecto de la durabilidad, funcionalidad, compatibilidad y seguridad, que presentan normalmente bienes del mismo tipo y que el consumidor pueda razonablemente esperar, dada la naturaleza de los bienes y teniendo en cuenta cualquier declaración pública realizada por el vendedor, o en su nombre, por otras personas en fases previas de la cadena de transacciones, incluido el productor, especialmente en la publicidad o el etiquetado.

Sin embargo, que la durabilidad pueda representar un criterio de conformidad subjetivo, y que sea siempre un criterio de conformidad objetivo, no cambia el hecho de que los plazos de responsabilidad sigan siendo los mismos; por ello, consideramos que la introducción de este criterio no supone ningún avance real en términos de sostenibilidad. 


\subsection{El sistema de garantías en concreto}

Para comprobar cuál es la aplicación práctica de la referencia que se hace en el mencionado considerando 32 de la Directiva a la economía circular, y en qué aspectos se manifiesta, si es que se manifiesta en alguno, analizaremos tres cuestiones fundamentales: los plazos de garantía, los plazos de inversión de la carga de la prueba y la jerarquía de los remedios.

\subsection{Los (escasos) plazos de garantía}

El artículo 10.1 DCB establece que el vendedor será responsable ante el consumidor por cualquier falta de conformidad que exista en el momento de la entrega del bien y se manifieste en el plazo de dos años a partir de ese momento. La normativa nueva ha decidido conservar así la duración del plazo establecida en la Directiva 1999/44/CE, aunque se permite que los legisladores nacionales contemplen garantías más extensas: el apartado tercero del artículo 10 explicita que "los Estados miembros podrán mantener o adoptar plazos más largos que los indicados".

Convendría tener en cuenta que la Propuesta original presentada por la Comisión en el año 2015 pretendía una armonización máxima también en lo relativo a los plazos fijados para las garantías legales; decisión que se justificaba en el considerando 32 de aquella Propuesta en los términos siguientes:

Para aumentar la seguridad jurídica de los vendedores y la confianza en general de los consumidores en las compras transfronterizas, es necesario armonizar el plazo durante el cual el vendedor será responsable de cualquier falta de conformidad que exista en el momento en que el consumidor adquiere la posesión material de los bienes. Considerando que la gran mayoría de los Estados miembros han previsto un plazo de dos años al implementar la Directiva 1999/44 y en la práctica los participantes del mercado lo consideran un plazo razonable, este plazo debe mantenerse.

Sin embargo, esta medida encontró críticas en la literatura académica por cuanto suponía una disminución de los derechos del consumidor; además, se trataba de una disposición poco coherente con los objetivos de sostenibilidad. Uno de los autores que dio la voz de alarma sobre esto fue $\operatorname{LOOS}(2017,21)$ quien entendía que el límite máximo de dos años no debería ser adoptado, porque en primer lugar, resultaría difícil justificar por qué el vendedor estaría exento de responsabilidad por falta de conformidad en bienes que el consumidor puede esperar razonablemente que duren más de dos años, como neveras, lavadoras o coches; $y$, en segundo lugar, la norma no constituiría incentivo alguno para que los productores produzcan bienes que duren más de dos años, haciendo que 
la competencia pudiese forzarles a alejarse de un enfoque de responsabilidad social corporativa.

La pretensión de armonización máxima en esta materia fue, por lo tanto, abandonada y ahora, de nuevo, seguirá tratándose de una cuestión que compete a los legisladores nacionales, que podrán mantener el plazo establecido por la DCB o ampliarlo. Como dice Terryn $(2019,134)$, el abandono de la armonización máxima sobre esta cuestión debe ser bien recibido; sin embargo, es desalentador que el legislador europeo no hubiese aprovechado la ocasión para incentivar una vida útil de los productos más larga, estableciendo unos plazos de garantía más extensos. No podemos estar más de acuerdo con tal afirmación, aunque todavía quedan razones para ser optimistas: puede (y sería aconsejable) que los legisladores nacionales decidan hacer uso de esta opción para extender los plazos de garantía: cuantos más Estados miembros así lo establezcan, más incentivos tendrán los productores para producir bienes que efectivamente sean más duraderos.

No cabe duda de que la ampliación de los plazos de garantía representa un mecanismo eficaz para transitar hacia una economía circular. Si bien como dice Pazos $(2020,214)$, cuánto más duradero sea un bien y más caro sea el precio a pagar, menos incentivos tendrán los consumidores para elegir los bienes sostenibles, tampoco es menos cierto que una mayor durabilidad podría erradicar ciertas prácticas contrarias a los objetivos de sostenibilidad, como la obsolescencia programada. Soto Pineda $(2015,8$ y ss.) lo explica así:

la relación entre la obsolescencia programada y la garantía -fuente de un régimen común adherido a la protección del consumidor-, se fundamenta, principalmente, en el protagonismo que las empresas que ponen en práctica la obsolescencia le dan al periodo de garantía (sea cual sea su determinación en un emplazamiento particular), erigiéndolo como uno de los elementos fundamentales que permiten planificar el tiempo y las circunstancias en las cuales los productos que fabrican devendrán obsoletos.

\subsubsection{La inversión de la carga de la prueba}

Según el artículo 11.1 DCB,

se presumirá que cualquier falta de conformidad que se manifieste en el plazo de un año a partir del momento de la entrega de los bienes, ya existía en el momento de la entrega de los bienes, salvo que se demuestre lo contrario o que esta presunción sea incompatible con la naturaleza de los bienes o con la índole de la falta de conformidad.

De nuevo, y una vez más, este precepto supone una excepción al principio de armonización máxima, pues el apartado segundo posibilita que Estados miembros 
mantengan o introduzcan "un plazo de dos años a partir de la fecha de entre de los bienes".

Esta disposición ha variado considerablemente respecto de la Propuesta original, en la que se había ampliado el plazo de inversión de la carga de la prueba de los seis meses (previsto en la Directiva 199/44/CE) a los dos años. Sin embargo, tal y como predijo LOOS $(2017,25)$, esta medida no fue finalmente incluida en la versión final, por tratarse de una cuestión políticamente controvertida. La ampliación de la inversión de la carga de la prueba se ha visto limitada a un año, dejando la opción a los Estados miembros de extenderlo hasta los dos años. No parece, del tenor literal del precepto, que sea posible, al menos en aquellos Estados miembros que prevén plazos de garantía más extensos, que los plazos de inversión de la carga de la prueba puedan equipararse y extenderse también consecuentemente. Esto no sólo repercute en la protección de los derechos de los consumidores, sino también en los objetivos de sostenibilidad: si el consumidor no consigue probar que un determinado bien no es conforme en el momento de la entrega de los bienes, no entrarán en funcionamiento los remedios legales previstos; hecho que tal vez le obligue a desechar el producto no conforme y comprar otro nuevo.

\subsubsection{La (insuficiente) jerarquía de remedios}

La Directiva (UE) 2019/771 mantiene un enfoque bastante conservador en lo relativo a la jerarquía de remedios previstos para la responsabilidad por falta de conformidad, pues a grandes rasgos, viene a ser igual que la establecida en la Directiva 1999/44/CE (Claeys \& Vancoillie, 2017, 178), aunque suavizada por la introducción de ciertas excepciones detalladas. La única gran diferencia es que con la nueva normativa, la jerarquía pasa a ser obligatoria para todos los sistemas nacionales (algunos no la habían previsto). Uno de los Estados miembros en los que no se prevé actualmente esa jerarquía es Portugal, por lo que una vez transpuesta la Directiva, los consumidores portugueses tendrán una protección menor; sin embargo, cierta doctrina portuguesa celebra la medida por considerarla más equilibrada y sostenible desde el punto de vida ambiental, por cuanto se prefiere la reparación frente a la resolución del contrato (Morais Carvalho, 2019, 81-82).

En efecto, esta jerarquía obligatoria de remedios debe ser bien recibida, en la medida en que puede incentivar el consumo sostenible y contribuir a una mayor durabilidad de los productos (Claeys \& Vancoillie, 2017, 178). Sin embargo, cabría preguntarse si las instituciones europeas no podrían haber dado un paso más allá, tal y como se cuestiona Terryn (2019, 127 y ss.). Para esta autora, que 
la reparación constituya un remedio primario es un acierto; sin embargo, lamenta que no sea suficientemente incentivada por la normativa europea, por no preferirse frente a la sustitución. La libertad de elección entre reparación o sustitución no concilia con los objetivos de sostenibilidad y lo deseable hubiera sido establecer una preferencia por la reparación. Además, en su opinión, la segunda "mejor opción" sería que la sustitución pueda hacerse con bienes reacondicionados. Tal práctica no se ha extendido demasiado, probablemente porque es controvertida desde la perspectiva de los derechos del consumidor. Sí es una práctica frecuente, al parecer, dentro de la compañía Apple, que ha tenido diversos procesos judiciales en Estados Unidos y los Países Bajos precisamente por sustituir dispositivos electrónicos por otros reacondicionados. En el caso de los Países Bajos, los tribunales han considerado que los consumidores tienen derecho al reemplazo por un bien nuevo; interpretación que es, en opinión de la doctrina (Terryn, 2019, 136; Mak \& Lujinovic, 2019, 172-173), correcta y comprensible desde la perspectiva de la protección del consumidor; sin embargo, se echa en falta que la argumentación jurídica tenga en cuenta la sostenibilidad, como sí lo hizo el Tribunal Supremo de Noruega en 2006 en un caso relativo a unas botas cuyos tacones se rompieron seis semanas después de la compra. En esta ocasión, el Tribunal Supremo decidió que la sustitución entrañaba costes no razonables y que la reparación debería ser suficiente, enfatizando, además, que era la opción más "ecofriendly" (Maitre-Ekern \& Dalhammar, 2019, 215-216).

\section{Perspectiva a corto plazo: ¿qué más se puede hacer?}

\section{I. Medidas por parte del legislador europeo}

Con mucha probabilidad, lo más importante para lograr una verdadera transición hacia la economía circular es informar y empoderar a los consumidores para que estos adopten patrones de consumo más sostenibles. Ya en el año 2012, y con motivo del Día mundial de los Derechos del Consumidor -que cada año se celebra el 15 de marzo y que se había dedicado, en aquella ocasión, al consumo sostenible--, el presidente del Comité Económico y Social Europeo afirmaba en su discurso de apertura que el consumo sostenible requiere que los consumidores jueguen un rol activo y esencial, y que por ese motivo, el trabajo de los Estados miembros es ayudarles:

Let us give them tools to make smart choices, to be better informed and to make well-reasoned decisions! Let us make sustainable products a real choice for consumers (Hobson, 2013, 1085-1086). 
La importancia de este objetivo es tal que requiere de una acción a gran escala por lo que, en nuestra opinión, sería necesario que las futuras reformas en esta materia provengan del legislador europeo.

Si se pretende, de verdad, enfatizar el papel de los consumidores como facilitadores de la economía circular (Keirsbilck \& Rousseau, 2019, 94), dos son las acciones más urgentes que debería llevar a cabo: de una parte, informarles mejor, y de otra, empoderarlos a través del reconocimiento de más o mejores derechos en relación con la durabilidad de los productos consumidos. Y es que como dice Pazos (2020, 215), los consumidores sólo entran en la ecuación de la circularidad en dos niveles: como beneficiarios de las obligaciones de información impuesta a los vendedores, y como sujetos de campañas de sensibilización dirigidas a que ellos demanden modelos sostenibles y circulares. Lamentablemente, las instituciones europeas han desaprovechado de forma reciente la oportunidad manifiesta de modificar estas dos cuestiones: con la aprobación de la Directiva (UE) 2019/2161, por la que se reforma la Directiva 211 /83/UE, se ha desperdiciado la ocasión de exigir deberes de información más específicos; y con la Directiva (UE) 2019/771, también se ha dejado de extender los plazos de garantía o reforzar el derecho a la reparación, tal y como ciertas organizaciones y autores reclamaban.

Pero todavía hay lugar para la esperanza: la Comunicación relativa a la Nueva Agenda del Consumidor, de 13 de noviembre de 2020, establece la transición ecológica como el primero de sus cinco ámbitos prioritarios. En este documento se destaca que los cambios de los patrones de consumo plantean nuevos desafíos, como el relativo al aumento de los residuos de envases de un solo uso, y que requiere "que se preste aún más atención a las iniciativas para apoyar la integración, la inclusión, la sensibilización y la educación de los consumidores". Los consumidores en Europea "muestran un creciente interés en contribuir personalmente a la consecución de la neutralidad climática", por lo que el reto consiste en aprovechar este potencial a través de medidas que permitan a cada consumidor

desempeñar un papel activo en la transición ecológica sin imponer un estilo de vida específico y sin ninguna discriminación social. El acceso a productos sostenibles no debe depender del nivel de ingresos o del lugar de residencia, sino que debe estar disponible para todos.

Los consumidores necesitan "información más fiable y de mayor calidad sobre los aspectos relacionados con la sostenibilidad de los bienes y servicios", pues "una mejor información sobre la disponibilidad de piezas de recambio y servicios de reparación puede aumentar aún más la durabilidad de los productos". Es necesario "otorgar a los consumidores un derecho efectivo de reparación". Además, 
se hace referencia a una futura revisión (a partir de 2022) de la Directiva sobre la compraventa de bienes, para lo que se

analizarán varias opciones relacionadas con las medidas correctoras disponibles para los consumidores, como dar preferencia a la reparación sobre la sustitución, ampliar el período de responsabilidad mínimo para los bienes nuevos o de segunda mano, reiniciar un nuevo período de responsabilidad tras la reparación.

\subsection{Medidas por parte de los legisladores nacionales}

Si la reciente respuesta del legislador europeo parece insatisfactoria, todavía cabe esperar que el legislador nacional sea más reactivo, con ocasión de la transposición de las Directivas 2019/770, 2018/771 y 2019/2161. Así, en una primera toma de contacto, las opciones más destacadas de las que dispone el legislador nacional para conciliar mejor la normativa de consumo con los planes para una economía circular son: (1) la ampliación generalizada de los plazos de garantía, (2) la ampliación de los plazos de garantía en función de la tipología de los bienes, y/o (3) el establecimiento de un nuevo período de garantía tras la reparación o sustitución. Todas estas alternativas no son excluyentes, sino que cabría su implementación de manera combinada.

En relación con la primera propuesta, cabe tener en cuenta que la ampliación de los plazos podría ser limitada o ilimitada. Una extensión limitada es lo que viene reclamando la ONG francesa Les Amis de la Terre desde hace tiempo, cuya campaña pretende extender el plazo de garantía de los productos de dos a diez años. En cuanto a la extensión ilimitada, cabría traer a colación los regímenes de Finlandia, Países Bajos, Irlanda o Reino Unido. En los dos primeros, no existe un límite en el plazo de garantía; en los dos últimos, no existe un plazo específico de garantía legal, pero los derechos de los consumidores están limitados por el plazo de prescripción. Que no exista límite en la garantía no quiere decir que se pueda presentar cualquier tipo de reclamación; como aclara Mańko $(2017,17)$, en estos sistemas, los tribunales desestimarán las demandas relativas a bienes perecederos, como flores o comida, o bienes baratos, que no aspiran a ser duraderos.

La segunda propuesta, relativa a la ampliación de los plazos en función de la tipología de los bienes, ya se recoge en ciertos países como Noruega, cuya normativa prevé un plazo general de dos años de garantía, que se extenderá hasta los cinco años para productos que se espera que duren más, como móviles, ordenadores, lavadoras, etc. (Maitre-Ekern \& Dalhammar, 2019, 215). Además,una medida de este calibre también está siendo objeto de debate en Austria, con motivo de la 
transposición de la Directiva (UE) 2019/771. Recientemente, y con ocasión del seminario "Digital Consumer Contract Law and New Technologies: Online Conference", celebrado los días 26 y 27 de noviembre de 2020, Brigitta Zöchling-Jud afirmaba que en Austria existe un borrador de transposición en el que se pretende la aprobación de una Ley de garantías del consumidor (que complementaría el Código Civil austríaco - ABGB) y en la que se aprecian varias propuestas sostenibles, toda vez que hay "por primera vez un gobierno que no sólo es conservador sino también verde": uno de ellos sería la imposición de un deber precontractual de informar acerca de la durabilidad mínima de bienes con elementos digitales o ropa textil; el otro, que los plazos de garantía se extenderían hasta los cinco años para los bienes que el consumidor pueda esperar que duren más de dos años. Aunque se trata, al parecer, de una propuesta políticamente controvertida, por lo que habrá que ver cómo se desarrolla finalmente la ley de transposición.

Por último, la tercera alternativa consiste en establecer un período de garantía adicional para el caso de que el bien haya sido reparado o sustituido. Esa obligación no sería una completa novedad en el plano nacional, pues según Mak \& Terryn $(2020,237)$ varios Estados Miembros ya prevén una garantía nueva después de la reparación o la sustitución (Austria, Croacia, Dinamarca, Estonia y Grecia), y algunos sólo prevén una nueva garantía en caso de sustitución (Hungría, Polonia, Portugal, Eslovaquía y España).

Otras opciones más discutibles que cabría plantearse, y que son de nuestra propia cosecha, serían: (1) la ampliación de los plazos de garantía sólo respecto de uno de los remedios o (2) el establecimiento de una presunción iuris tantum posibilitando la reparación si el producto deja de funcionar tras la expiración del plazo de garantía. La primera propuesta consistiría en combinar la posibilidad que tienen los Estados miembros de ampliar los plazos de garantía, con la reivindicación que hacen alguos autores acerca de la preferencia que debería dársele a la reparación frente a la sustitución. Se trataría, pues, de ampliar los plazos de garantía pero únicamente respecto de uno de los remedios: el de reparación. Así, si uno compra un bien, gozará de todos los remedios disponibles durante el plazo general de garantía (supongamos, de dos años), pero si el bien dejase de ser conforme posteriormente, todavía podría pedir la reparación (imaginemos, por ejemplo, durante los cinco años siguientes a la compra). También relacionado con esto estaría la segunda propuesta, relativa a establecer una presunción iuris tantum de que si un bien o producto deja de funcionar o se vuelve defectuoso al poco tiempo de terminar el plazo de garantía, es porque se ha diseñado deliberadamente su caducidad prematura y, por lo tanto, todavía tendrá el consumidor derecho a la reparación. En este último caso, no resulta del todo claro que el legislador nacional pudiese 
establecer una medida de tal naturaleza, debido a que la Directiva (UE) 2019/771 es de armonización máxima. Se podría argumentar, en un sentido afirmativo, que la propia Directiva determina que regula "determinados aspectos de los contratos de compraventa" y que, por lo tanto, los legisladores nacionales podrán legislar acerca de las cuestiones no expresamente contempladas en esta Directiva.

En un segundo plano de iniciativas, cabría considerar la posibilidad de regular la transferabilidad de la garantía en caso de bienes de segunda mano, tal y como propone LOOS (2017, 49-50). Con el auge del mercado de segunda mano, a través de la compraventa en ciertas plataformas online, esta cuestión gana importancia, ya que es improbable que el comprador original esté dispuesto a invocar los remedios bajo la garantía en representación del segundo comprador, si el segundo comprador no puede ejercer esos remedios por sí mismo. Debe tenerse en cuenta que, tal y como señala el autor, algunas asociaciones de consumidores remitieron comentarios al Libro Verde sobre la revisión del acervo en materia de consumo en favor de una transferencia automática de esos derechos; además, el artículo IV.A, 6:102(2) del Proyecto de Marco Común de Referencia establece que

If not otherwise provided in the guarantee document, the guarantee is also binding without acceptance in favour of every owner of the goods within the duration of the guarantee;

por último, la Propuesta original de la Directiva sobre los derechos de los consumidores de 2008 también consideraba como abusiva toda cláusula contractual que restringiese "el derecho del consumidor a revender los bienes al limitar la transferibilidad de las garantías comerciales ofrecidas por el comerciante". Sin embargo, ni en el CESL (Propuesta de Reglamento relativo a una normativa común de compraventa europea del año 2011 1), ni las dos Directivas recientemente aprobadas (770/2019 y 771/2019) incluyen disposición alguna al respecto; por ello, Loos considera que ya es hora de que se avance en esta cuestión.

Lo que sí puede hacer el legislador nacional, en sentido negativo, es no alterar el plazo de garantía para los bienes de segunda mano, tal y como posibilita el artículo 10.6 DCB:

Los Estados miembros podrán disponer que, en el caso de los bienes de segunda mano, el vendedor y el consumidor puedan convenir términos contractuales o pactos con un período de responsabilidad o plazo de prescripción más breve que los previstos en los apartados 1, 2 y 5 [de dos años], siempre que ese período o plazo más breve no sea inferior $a$ un año.

Aunque también cabe tener en cuenta que, para Pazos $(2020,215)$, no está del todo claro que un mayor período de garantía para los bienes de segunda mano 
contribuya a un modelo más circular pues, en ese caso, los consumidores estarían más dispuestos a comprar bienes de segunda mano, pero sólo si no hubiese otros efectos colaterales. Si la responsabilidad aumenta, los vendedores de bienes de segunda mano podrían pagar menos para adquirir bienes usados, adquirir sólo bienes que estén en muy buenas condiciones, o incrementar el precio de la reventa.

En definitiva, no se deben subestimar las diversas opciones normativas de las que disponen los legisladores nacionales para poner su grano de arena en la transición hacia la economía circular. En el caso de España, parece que existe actualmente la voluntad de crear normas para contribuir a una mayor sostenibilidad; sin embargo, la conexión con el Derecho de consumo es débil todavía (Pazos, 2020, 213). Esta puede ser una buena oportunidad para dar valor a la Estrategia "España Circular 2030", aprobada en junio de 2020, donde se establece, como sector prioritario de actuación, el de los bienes de consumo. En este sentido, cabe tener en cuenta que exite ya un Anteproyecto de Ley (no definitivo) por el que se modifica el TRLGDCU, con objeto de transponer al ordenamiento jurídico español las Directivas (UE) $2019 / 770,2019 / 771$ y $2019 / 2161$. Entre los puntos positivos a resaltar, cabría mencionar la inversión de la carga de la prueba, que se amplía hasta los 2 años (artículo 121), o que durante el año posterior a la entrega del bien ya conforme, el empresario respondería de las faltas de conformidad, tanto si el bien hubiese sido reparado como sustituido (artículo $122.3^{\circ}$ ). Es también positivo, de alguna manera, que el artículo 120 prevea una ampliación de la responsabilidad por bienes en soporte físico a 3 años, aunque sin lugar a dudas, se podría haber ido más allá. Finalmente, cabe mencionar que este mismo artículo 120 permitiría pactar un plazo menor de responsabilidad para los bienes de segunda mano, que no podría ser inferior a un año desde la entrega.

\subsection{La autorregulación del mercado}

Desde los primeros días en que surgen las políticas medioambientales ha existido tensión entre el propósito de protección del medio ambiente y el reclamo de las sociedades de crecimiento y prosperidad (Schally, 2019, 3). Sin embargo, ambos objetivos no deben considerarse divergentes, ya que cabe esperar que el mercado se vaya autodirigiendo hacia la circularidad. Los cambios que afectan a nuestra sociedad en general, y de forma muy concreta, la digitalización, pueden conseguir importantes cambios en los patrones de consumo como ocurre, por ejemplo, con la economía o las plataformas colaborativas. En los últimos tiempos también ha adquirido especial relevancia la servitización, por la que no se ofrece la venta de un bien, sino un servicio con uso del bien, o los "product-service systems" o PSS, 
en los que no sólo se ofrece un producto, sino también un servicio aparejado (por ejemplo, el de mantenimiento/reparación). Cualquiera de estos nuevos modelos económicos se consideran y publicitan como más ecosostenibles, pues contribuyen a una mayor durabilidad y reparabilidad, prolongando el "service-life" de los productos (Keirsbilck \& Rousseau, 2019, 106 y ss.).

Existen, también, importantes iniciativas empresariales cuyos objetivos económicos no compiten con los medioambientales, sino todo lo contrario. Cabe destacar, por ejemplo, el caso de ReTuna, un centro comercial sueco donde todas las tiendas venden cosas usadas (Maitre-Ekern \& Dalhammar, 2019, 218); o el de Mud Jeans en los Países Bajos, en el que el consumidor no compra un pantalón, sino que lo alquila por doce meses. Con esta iniciativa se consigue evitar la obsolescencia psicológica o de estilo, a través del retorno de un producto después de un determinado tiempo de uso (Mak \& Lujinovic, 2019, 176). Además, se ha afirmado por la doctrina que cada vez existe una mayor demanda por productos que respeten el medio ambiente (Mak \& Terryn, 2020, 228) así que no debe causar sorpresa que, a medida que los consumidores se vuelven más sensibles al impacto medioambiental de los productos y servicios, los negocios sean más proclives a hacer declaraciones explícitas e implícitas sobre el impacto de sus productos y servicios (Keirsbilck \& Rousseau, $2019,105)$. Y tal vez sea aquí donde haya mayor lugar para la esperanza, porque cuanta más demanda exista en términos de sostenibilidad, mayor será la calidad ofertada. Las garantías comerciales pueden representar, en este sentido, un instrumento ideal para estimular la reparación y, por ende, la durabilidad de los productos (Mak \& Lujinovic, 2019, 169); aunque esto no siempre será así, por ejemplo, en los casos en los que la empresa establece una garantía de reemplazo directa (Terryn, 2019, 137). Cabe lamentar, en este sentido, que la propuesta de Tonner \& Malcolm (2017, 28 y ss.), relativa a la imposición de un deber a cargo de los vendedores de ofrecer una garantía comercial indicando la vida útil de un determinado producto, no fuese tenida en cuenta por el legislador europeo para su inclusión en la Directiva (UE) 2019/771.

\section{Conclusiones}

El ritmo vertiginoso de sobreconsumismo que se observa en la sociedad actual es alarmante, y por ello, todas las medidas que se puedan tomar probablemente corran el riesgo de ser insuficientes a la hora de frenar las consecuencias medioambientales que de aquél se deriven. El problema más acuciante en el momento presente es la inercia; por ello, es necesario repensar la economía, replantearse la sociedad 
en la que queremos vivir y abrir un debate teórico sobre cuál debe ser el rol que asuma el Derecho (Micklitz, 2019, 325 y 345). Derecho que, como ciencia social comportamental que es, puede contribuir a las transformaciones deseadas (Ribeiro Freyesleben y Maas Dos Anjos, 2018, 826). Resulta obvio, entonces, que para alentar la transición hacia una economía circular, los gobiernos necesitan crear las condiciones que estimulen y aceleren las decisiones en este sentido (De Boeck, 2019, 186-187).

La construcción de una economía circular va más allá del Derecho de consumo, en el sentido de que se necesitan medidas en un amplísimo abanico de sectores (Keirsbilck \& Rousseau, 2019, 94; Mak \& Terryn, 2020, 229); sin embargo, en este trabajo hemos podido analizar cuán desalentadora es la deficiente aproximación de la normativa europea de consumo hacia la circularidad. Como dicen Mak \& Terryn $(2020,230)$, la poca literatura que ha estudiado la relación entre el Derecho de consumo y la sostenibilidad revela que la política europea se ha limitado a un régimen de "light touch", que apela a los ciudadanos a transitar hacia estilos de vida más sostenibles, sin forzarles a cambiar su comportamiento o a reducir su consumo. Especialmente decepcionante resulta que no se hubiesen aprovechado las recientes iniciativas legislativas (Directivas 2019/770, 2019/771 y 2019/2161) para abordar una reforma de calado desde una perspectiva medioambiental.

Pero, como hemos visto, todavía hay margen para el cambio: los legisladores nacionales dispondrán de la posibilidad de dar un paso más con sus normas de transposición, a través, por ejemplo, de la ampliación de los plazos de garantía. Además, también está previsto una futura revisión europea de las normativas anteriormente mencionadas, y cabe esperar, debido a la mayor demanda de productos sostenibles, que el mercado termine autodigiriéndose hacia modelos económicos con menor impacto medioambiental. Debemos confiar, pues, en el pronóstico del Informe de la Comisión de 2019, sobre la aplicación del Plan de Economía Circular de 2015, cuando afirma que "la economía circular es ahora una megatendencia mundial irreversible".

\section{Bibliografía}

Bauman, Z. (2006). Vida líquida. Barcelona: Paidós.

Claeys, I. \& Vancoille, J. (2017). "Remedies, modification of digital content and right to terminate long-term digital content contracts". En I. CLAEYS \& E. TeRrYN 
(Eds.) Digital content \& distance sales: new developments at EU level. Cambridge: Intersentia, 167-232.

De BoeCK, A. (2019). "The 'Circular Economy' - National Legal Initiatives and Challenges Converning Movables - Belgium". En B. KeIRSBILCK \& E. TeRRYN (Eds.) Consumer Protection in a Circular Economy. Cambridge: Intersentia, 185-199.

HOBSON, K. (2013). "Weak or strong sustainable consumption? Efficiency, degrowth, and the 10 Year Framework of Programmes". Environment and Planning C: Government and Policy, 31, 1082-1098.

Howells, G., TwigG-Flesner, C. \& Wilhelmsson, T. (2018). "Regulation of unfair commercial practices". En Rethinking EU Consumer Law. Oxon-New York: Routledge, 46-93.

KeIRSBILCK, B. \& Rousseau, S. (2019). "The Marketing Stage: Fostering Sustainble Consumption Choices in a Circular and Functional Economy". En B. KeIRSBILCK \& E. TERRYN (Eds.) Consumer Protection in a Circular Economy. Cambridge: Intersentia, 93-126.

LIBAERT, T. \& HABER, J. P. (2013). Por un consumo más sostenible: la duración de la vida de los productos industriales y la información al consumidor para recuperar la confianza: la duración de la vida de los productos industriales y la información al consumidor para recuperar la confianza. Bruselas: Dictamen del Comité Económico y Social.

Loos, M. B. M. (2017). "Not good but certainly content: The proposals for European Harmonisation of Online and Distance Selling of Goods and the Supply of Digital Content". En I. ClaeYs \& E. TeRrYN (Eds.) Digital content \& distance sales: new developments at EU level. Cambridge: Intersentia, 3-53.

Maitre-Ekern, E. \& Dalhammar, C. (2019). "A Scandinavian Perspective on the Role of Consumers in the Circular Economy". En B. KeIRSBILCK \& E. TerryN (Eds.) Consumer Protection in a Circular Economy. Cambridge: Intersentia, 201-223.

MAK, V. \& LUINNOVIC, E. (2019). "Towards a Circular Economy in EU Consumer Markets. Legal Possibilities and Legal Challenges and the Dutch Example". En B. KeIRSBILCK \& E. TerRYN (Eds.) Consumer Protection in a Circular Economy. Cambridge: Intersentia, 161-183. 
Mak, V. \& Terryn, E. (2020). "Circular Economy and Consumer Protection: The Consumer as a Citizen and the Limits of Empowerment Through Consumer Law". Journal of Consumer Policy, 43, 227-248.

MAK, V. (2016). The new proposal for harmonised rules on certain aspects concerning contracts for the supply of digital content". Brussels: Directorate-General for Internal Policies, European Parliament.

MAŃKO, R. (2017). Towards new rules on sales and digital content: Analysis of the key issues". Brussels: European parliamentary Research Service.

MAZEIKA, R. (2019). "Consumer Protection in the Circular Economy in Europe- Legal Initiatives and Challenges - The Commission's View". En B. KEIRSBILCK \& E. TERRYN (Eds.) Consumer Protection in a Circular Economy. Cambridge: Intersentia, 11-18.

Mesacasa, A, Corona, H. M. P. \& Mello, N. A. (2010). "Ecodesign: um Novo Paradigma Moderno Inscrito entre Moda e Consumo?". Design \& Tecnologia, 1(2), 1-7.

MickLtz, H.-W. (2019). "Squaring the Circle? Reconciling Consumer Law and the Circular Economy". En B. KeIRSBILCK \& E. TeRrYN (Eds.) Consumer Protection in a Circular Economy. Cambridge: Intersentia, 323-345.

Morals Carvalho, J. (2019). "Venda de bens de consume e fornecimento de conteúdos e serviços digitais - as Diretivas 2019/771 e 2019/770 e o seu impacto no Direito portugués". Revista electrónica de Direito, 20 (3), 63-87.

Nascimento Pereira, M. (2017). "Consumo sustentável: a problemática da obsolescência programada e o descarte de produtos". Revista Eletrônica Direito e Sociedade, 5 (2), 209-220.

Norma Martínez, A. y Margarita Porcell, A. (2016). "Un difícil camino en pos del consumo sustentable: el dilema entre la obsolescencia programada, la tecnología y el ambiente". Lex: Revista de la Facultad de Derecho y Ciencia Política de la Universidad Alas Peruanas, 14 (18), 333-378.

Pantzar, M. \& Sulada, T. (2020). Delivering a circular economy within the planet's boundaries: An analysis of the new EU Circular Economy Action Plan. Brussels and Stockholm: Institute for European Environmental Policy (IEEP) and Stockholm Environment Institute (SEI). 
Pazos, R. (2020). "Sustainability, the Circular Economy and Consumer Law in Spain". Journal of European Consumer and Market Law, 5, 21 2-215.

Ribeiro Freyesleben, L. E. y MaAs Dos Anjos, R. (2018). "Circularidad en tiempos obsoletos". En J. Melgarejo Moreno (Ed.) Congreso Nacional del Agua Orihuela: Innovación y Sostenibilidad. Alicante: Universitat d'Alacant, 819-830.

Santamaría Arinas, R. J. (2019). "Economía circular: líneas maestras de un concepto jurídico en construcción". Revista Catalana de Dret Ambiental, 10 (1), 1-37.

Schally, H. M. (2019). "The Circular Economy Action Plan: An Agenda for Change". En B. KelRSBILCK \& E. TeRRYN (Eds.) Consumer Protection in a Circular Economy. Cambridge: Intersentia, 3-9.

Soto PINEDA, J. A. (2015). "Reflexiones acerca de las posibles incompatibilidades de la obsolescencia programada con el sistema de defensa de los consumidores". Actualidad civil, 6, 40-55.

Straetmans, G. \& Meys, S. (2017). "The new proposals for directives concerning digital content and online/distance sales". En I. CLAEYS \& E. TeRrYN (Eds.) Digital content \& distance sales: new developments at EU level. Cambridge: Intersentia, 307-361.

TeRRYN, E. (2019). "A Right to Repair? Towards Sustainable Remedies in Consumer Law". En B. KeIRSBILCK \& E. TerRrN (Eds.) Consumer Protection in a Circular Economy. Cambridge: Intersentia, 127-147.

TONNER, K. \& MALCOLM, R. (2017). How an EU Lifespan guarantee Model Could Be Implemented Across the European Union. Brussels: European Parliament.

VIDALENC, E. y MEUNIER, L. (2014). "Obsolescence des produits: I'impact écologique". Revue Futuribles, 402, 5-21. 